\title{
Improved synthetic spectra of helium-core white dwarf stars
}

\author{
R. D. Rohrmann, ${ }^{1 \star} \dagger \ddagger$ A. M. Serenelli, ${ }^{2 \star} \S$ L. G. Althaus ${ }^{2 \star}$ and O. G. Benvenuto ${ }^{2 \star} \|$ \\ ${ }^{1}$ Observatorio Astronómico, Universidad Nacional de Córdoba, Laprida 854, (5000) Córdoba, Argentina \\ ${ }^{2}$ Facultad de Ciencias Astronómicas y Geofísicas, Universidad Nacional de La Plata, Paseo del Bosque S/N, (1900) La Plata, Argentina
}

Accepted 2002 May 1. Received 2002 May 1; in original form 2002 March 14

\begin{abstract}
We examine the emergent fluxes from helium-core white dwarfs following their evolution from the end of pre-white dwarf stages down to advanced cooling stages. For this purpose, we include a detailed treatment of the physics of the atmosphere, particularly an improved representation of the state of the gas by taking into account non-ideal effects according to the so-called occupation probability formalism. The present calculations also incorporate hydrogen-line opacity from Lyman, Balmer and Paschen series, pseudo-continuum absorptions and new updated induceddipole absorption from $\mathrm{H}_{2}-\mathrm{H}_{2}, \mathrm{H}_{2}-\mathrm{He}$ and $\mathrm{H}-\mathrm{He}$ pairs. We find that the non-ideal effects and line absorption alter the appearance of the stellar spectrum and have a significant influence upon the photometric colours in the UBVRI-JHKL system. This occurs specially for hot models $T_{\text {eff }} \gtrsim 8000$ owing to line and pseudo-continuum opacities, and for cool models $T_{\text {eff }} \lesssim 4000$ where the perturbation of atoms and molecules by neighbouring particles affects the chemical equilibrium of the gas. In the present study, we also include new cooling sequences for heliumcore white dwarfs of very low mass $\left(0.160\right.$ and $\left.0.148 \mathrm{M}_{\odot}\right)$ with metallicity $Z=0.02$. These computations provide theoretical support to search for and identify white dwarfs of very low mass, specially useful for recent and future observational studies of globular clusters, where these objects have began to be detected.
\end{abstract}

Key words: atomic processes - stars: atmospheres - stars: evolution - white dwarfs.

\section{INTRODUCTION}

It is generally accepted that helium-core white dwarf (He WD) stars constitute the final product of the evolution of some close binary systems. This is so because an isolated star would need a time-scale much longer than the present age of the Universe to reach a $\mathrm{He}$ WD configuration. The mass of these objects should be smaller than that required for degenerate helium ignition, $\approx 0.45 \mathrm{M}_{\odot}$ (Mazzitelli 1989), thus resulting in very-low-mass WDs. Low-mass WDs have been detected in large surveys (Bragaglia et al. 1990; Bergeron, Saffer \& Liebert 1992; Bragaglia, Renzini \& Bergeron 1995; Saffer,

\footnotetext{
^E-mail: rohr@astroscu.unam.mx (RDR); serenell@fcaglp.fcaglp.unlp.edu. ar (AMS); althaus@fcaglp.fcaglp.unlp.edu.ar (LGA); obenvenu@fcaglp. fcaglp.unlp.edu.ar (OGB)

$†$ Present address: Instituto de Astronomía, UNAM, A.P. 70-264, 04510 México D.F., México.

$\ddagger$ Postdoctoral Fellow of the IA-UNAM.

$\S$ Fellow of the Consejo Nacional de Investigaciones Científicas y Técnicas (CONICET), Argentina.

\Member of the Carrera del Investigador Científico y Tecnológico, CONICET, Argentina.

\|Member of the Carrera del Investigador Científico, Comisión de Investigaciones Científicas de la Provincia de Buenos Aires, Argentina.
}

Livio \& Yungelson 1998) and represent an appreciable fraction of the total population of WD stars.

The interest in the study of these stars has greatly increased since their binary nature was placed on a solid observational ground by Marsh (1995) and Marsh, Dhillon \& Duck (1995). Indeed, He WDs have been detected in numerous binary systems containing usually either another WD or a millisecond pulsar (Moran, Marsh \& Bragaglia 1997; Orosz et al. 1999; van Kerkwijk et al. 2000, amongst others). Such systems are very interesting because they enable us to infer the properties of one component of the system from studying the physical properties of the other. This is important in connection with pulsar mass determinations and the structure and evolution of WD stars.

The recent detection of low-mass He WDs $\left(M \lesssim 0.20 \mathrm{M}_{\odot}\right)$ in compact binaries belonging to globular clusters (Cool et al. 1998; Edmonds et al. 1999; Taylor et al. 2001; Edmonds et al. 2001) has also sparked the attention of many researchers. The interest in studying He WDs in globular cluster is motivated not only by their importance in the understanding of the formation and evolution of the compact binaries in which these stars are found but also by the possibility they offer of constraining globular cluster dynamics and evolution.

Numerous studies have been devoted to the evolution of He WDs. Amongst them we mention those of Benvenuto \& Althaus (1998), 
Driebe et al. (1998), Hansen \& Phinney (1998), Sarna, Antipova \& Ergma (1999) and Althaus, Serenelli \& Benvenuto (2001). In particular, Althaus et al. (2001) have investigated the effect of element diffusion on the evolution of He WDs taking into account the evolutionary stages prior to the WD formation. These authors find that element diffusion induces thermonuclear hydrogen shell flashes in $\mathrm{He}$ WD models with stellar masses in the range $0.18 \lesssim M / \mathrm{M}_{\odot} \lesssim 0.41$. In particular, Althaus et al. (2001) show that the occurrence of such diffusion-induced flashes leads to He WD models with hydrogen envelope masses too small to support any further nuclear burning, thus implying much shorter cooling ages than in the case when diffusion is neglected. As shown by Althaus et al. (2001), such short cooling ages remove the discrepancy between the spin-down age of the millisecond pulsars B1855 + 09, PSR J0034 - 0534 and PSR $\mathrm{J} 1012+5307$ and the cooling ages of their He WD companions. Evolutionary calculations of Althaus et al. (2001) have received tentative support from the optical detection of the WD companion to the millisecond pulsar in 47 Tucanae (Edmonds et al. 2001).

Colours and magnitudes appropriate for old He WDs with the predictions of stellar evolution and element diffusion have recently been derived in a self-consistent way by Serenelli et al. (2001). In the Serenelli et al. (2001) calculations, emphasis is placed on the late stages of evolution where WD cooling is strongly affected by the treatment of the outer layers, particularly of the atmosphere. These authors have explored at some length the evolution of He WDs and found that when the effective temperature decreases below $4000 \mathrm{~K}$, the emergent spectrum of these stars becomes bluer within timescales of astrophysical interest. Because Serenelli et al. (2001) were interested in the late stages of He WD evolution, they did not attempt a detailed modeling of the emergent spectrum of these stars at high effective temperature stages $\left(T_{\text {eff }} \gtrsim 8000 \mathrm{~K}\right)$.

In this paper we improve the calculations of Serenelli et al. (2001) by including a more detailed treatment of the microphysics entering the WD atmosphere. This enables us to derive accurate colours and magnitudes for He WDs at high effective temperatures where the effects of line broadening opacities are not negligible. Another aim of the present work is to extend the evolutionary calculations presented in Serenelli et al. (2001) to less-massive He WD models on the basis of a more physically sound treatment of the mass transfer stage than that attempted in Serenelli et al. (2001). This will allow us to compare the predictions of our evolutionary models with the observations of the He WD candidates recently reported in the globular clusters NGC 6397 and 47 Tucanae by Taylor et al. (2001) and Edmonds et al. (2001), respectively.

The magnitude calculations in the ultraviolet and optical regions are strongly sensitive to the inclusion of hydrogen-line opacity (particularly for $T_{\text {eff }} \gtrsim 8000 \mathrm{~K}$ ), which has been neglected in our previous model grids (Rohrmann 2001; Serenelli et al. 2001). For the present work, we include this additional opacity source in the atmosphere code and we re-calculate the models of Serenelli et al., yielding a better prediction of the colour indices for hot He WDs. Furthermore, the evaluation of non-ideal effects in the gas allows us to calculate more realistic atmosphere models for WDs at both early and advanced stages of cooling. In fact, WD atmospheres are well known to be characterized by high densities (as high as $\rho \approx 0.1 \mathrm{~g} \mathrm{~cm}^{-3}$ ), where interactions amongst neighbouring particles, charged and neutral, affect the number of bound states of atoms and molecules. These particle perturbations can modify the chemical equilibrium of the gas and its optical properties. The occupation probability formalism of Hummer \& Mihalas (1988) provides a tool to treat non-ideal effects in the gas of stellar envelopes. This theory avoids typical rough truncations of particle partition functions giving a thermodynami- cally self-consistent way to calculate the ionization and excitation equilibrium in the gas. The occupation formalism was introduced by Bergeron (1988) in the context of WD atmospheres and we have chosen it for the present work.

The application of Hummer-Mihalas formalism for calculating atomic populations necessitates corresponding improvements in the formulae used to compute the optical properties of the gas. Phenomenological proposals of simulations of the optical spectra in the framework of the occupational formalism have been given by Däppen, Anderson \& Mihalas (1987) and Hubeny, Hummer \& Lanz (1994). These researchers introduce the calculation of the so-called pseudo-continuum absorption, which arises from the ionization of strongly-perturbed atoms. This opacity source becomes very important in WD stars for different ranges of effective temperatures, particularly when the Balmer lines contribute significantly to the total opacity (e.g. Bergeron, Wesemael \& Fontaine 1991). In view of the preceding considerations, we found it necessary to adopt here a particular optical representation for our atmosphere calculations. It is worth noting that the opacity theory for non-ideal gases is still in a preliminary stage and additional studies are necessary.

In addition to the improvements perfomed on the equation of state of the gas, new quantum mechanical data of collision-induced absorption (CIA) processes have been incorporated in our atmosphere code. These new data yield significant changes in the emergent radiative energy obtained for cool He WD models as compared with previous calculations.

The paper is organized as follows: Section 2 describes the new treatments of the input physics in the atmosphere code. In Section 3, the atmosphere models are coupled with evolutionary calculations and news, accurate results are given for very-low-mass He WD stars. Conclusions are presented in Section 4.

\section{MODEL ATMOSPHERES}

The model atmospheric structures were computed by means of a code developed recently (Rohrmann 2001), which has been improved in a number of aspects. In what follows, we describe at some length the main improvements we have included in our code.

\subsection{Non-ideal effects in the gas equation of state}

One of the most important improvements considered in this work concerns the incorporation in our code of the occupational probability formalism of Hummer \& Mihalas (1988) for the treatment of non-ideal effects in the gas equation of state. This formalism is based on the free-energy-minimization technique (cf. Graboske, Harwood \& Rogers 1969) and can be synthesized by writing, for each chemical species, the LTE (local thermodynamic equilibrium) population of an internal state $i\left(n_{i}\right)$ relative to the total particle density $\left(n_{\mathrm{tot}}\right)$ of this species as

$\frac{n_{i}}{n_{\mathrm{tot}}}=\frac{w_{i} g_{i} \exp \left(-E_{i} / k T\right)}{Z}$,

where $w_{i}$ is the so-called occupation probability of level $i, g_{i}$ the statistical weight, $E_{i}$ the excitation energy, $Z$ the internal partition function, $k$ the Boltzmann constant and $T$ the gas temperature.

Factors $w_{i}$ are given by partial derivation of a non-ideal term $f$ in the Helmholtz free energy of the gas,

$w_{i}=e^{\left(-\partial f / \partial n_{i}\right) / k T}$.

The probabilistic interpretation of $w_{i}$ allows Hummer and Mihalas to combine the action of statistically independent interactions. They have considered two types of perturbation. One of them is the 
excluded volume interaction (also called perturbation by neutral particles), which arises from the finite size of particles with bound electronic states. These perturbations are taken into account through the second virial coefficient in the van der Waals equation of state, which implies an occupation probability of the form

$w_{i}($ neutral $)=\exp \left[-\frac{4 \pi}{3} \sum_{j} n_{j}\left(r_{i}+r_{j}\right)^{3}\right]$,

where the sum runs over all species $j$ having bound levels, $r_{i}$ and $r_{j}$ being the effective interaction radii of involved particles.

The other type of perturbation of atomic levels is due to charged species. In the Hummer-Mihalas formalism, these perturbations are calculated from a fit to a quantum mechanical Stark ionization theory. This yields an occupation probability that can be formally expressed as

$w_{i}($ charged $)=Q(\beta)$,

which is a fit over the distribution of the electric field strength $\beta$ arising from the charged particles. Originally, the Holtsmark distribution was adopted as the micro-field distribution function. However, this distribution does not take into account the correlations of the charged perturbers. A more appropriated distribution, which includes correlation effects, was presented by Nayfonov et al. (1999). The numerical fits given by Nayfonov et al. to compute $w_{i}$ (charged) have been adopted in our calculations. Finally, assuming statistically independent perturbations, the joint occupation probability of an atomic state is the product of $w_{i}$ (neutral) and $w_{i}$ (charged).

In our calculations the atmospheric gas is assumed to be composed of 'bound' species (particles with electronic structure) $\mathrm{H}, \mathrm{H}_{2}$, $\mathrm{H}^{-}, \mathrm{H}_{2}^{+}, \mathrm{H}_{3}^{+}, \mathrm{He}$ and $\mathrm{He}^{+}$, and 'bare' particles $\mathrm{H}^{+}, \mathrm{He}^{++}$and $\mathrm{e}^{-}$. The energy levels for bound particles have been chosen to be those of the isolated atom or molecule. Eigenenergy shifts could be expected as the density is raised. However, according to experimental measurements (e.g. Weise, Kelleher \& Paquette 1972), unperturbed energy levels are a good approximation at low densities $(\log \rho \lesssim-2)$ and seem reasonable at higher densities (Saumon \& Chabrier 1991).

Characteristic atomic and molecular radii are required in order to evaluate the excluded volume perturbations. The equivalent hardsphere radii (in $\AA$ ) for $\mathrm{H}_{2}$ and $\mathrm{H}$ are computed from linear fits to results of Saumon \& Chabrier (1991, fig. 6):

$r_{\mathrm{H}}=1.876-0.294 \log T+(-0.576+0.100 \log T) \rho$,

$r_{\mathrm{H}_{2}}=2.226-0.331 \log T+(-0.975+0.175 \log T) \rho$,

which are based on a temperature- and density-dependent thermodynamic criterion (Weeks, Chandler \& Andersen 1971). Radii for excited states are obtained by simple scaling laws.

The molecular hydrogen $\mathrm{H}_{2}$ is represented by 193 vibrationrotational states with energy values calculated from the expression given by Herzberg (1950), which includes anharmonicity corrections, deviations from the rigid rotator approximation, and vibration-rotation coupling. Excited electronic states of $\mathrm{H}_{2}$ are above the ground state by more than $11 \mathrm{eV}$, so they can be safely neglected. Effective radii for vibration-rotational states were calculated following Mihalas, Däppen \& Hummer (1988), i.e. the equations (11) and (12) of Vardya (1965) were solved for each state. These radii were then scaled to ground radius (equation 6).

The neutral helium is represented by $144(n, L, S)$ states $(1 \leqslant n \leqslant 50)$, with energy values for $n<22$ based on the data of Martin (1973). For $n>22$, energies (in $\mathrm{eV}$ ) relative to ground level were estimated with the expression

$E_{n}=24.5876-1.002866 \frac{\chi_{H}}{n^{2}}$,

where $\chi_{H}$ is the ionization potential of the atomic hydrogen. Equation (7) is a fit to Martin's data for $15<n<23$ and its extrapolation to $n>22$ has an estimated error lower than $10^{-3} \mathrm{eV}$. Effective radii of the excited states of He were evaluated from the hydrogenic formula (performing the calculation of the expectation value of $r$ with the well-known electronic wave functions)

$\langle r\rangle_{(n, l)}=\frac{r_{o}}{3}\left[3 n^{2}-l(l+1)\right]$,

which is normalized so that the ground-state radius is $r_{o}=3 a_{0} / 2 Z_{a}$ ( $a_{0}$ is the Bohr radius and $Z_{a}$ the atomic number). The expression (8) yields a value of $0.40 \AA$ for the radius of both $\mathrm{He}$ and $\mathrm{He}^{+}$ ground states. However, we can expect that the He radius is greater than the $\mathrm{He}^{+}$radius owing to its smaller binding energy. Therefore, we adopted a radius of $0.50 \AA$ for the He ground state, as used by Mihalas et al. (1988). We rescaled the radii of the He excited states according to this $r_{o}$ value.

For atomic hydrogen we used 100 energy levels calculated in the standard form, and the effective radii were evaluated using the expression (8) averaged over the azimuthal quantum number $l$,

$\langle r\rangle_{(n)}=\frac{r_{o}}{6}\left(5 n^{2}+1\right)$,

with $r_{o}$ given in equation (5). Similarly, $\mathrm{He}^{+}$was represented by 250 hydrogenic energy levels having radii defined by equation (9) with $r_{o}=3 a_{0} / 2 Z_{a}=0.40 \AA$.

Following Lenzuni \& Saumon (1992), we have chosen as characteristic radii of $\mathrm{H}^{-}, \mathrm{H}_{2}^{+}, \mathrm{H}_{3}^{+}$the values $1.15,1.32$ and $0.65 \AA$, respectively. For each of these species we assigned an occupation probability corresponding to a representative state with an energy given by the ionization or dissociation energy of the considered particle.

In the frame of the occupation probability formalism, the ideal gas equation of state (EOS) which relates pressure, temperature and density, must be corrected taking into account the non-zero size of the molecules and atoms. A simple interpolation formula that satisfies this requirement follows from the van der Waals's equation (e.g. Landau \& Lifshitz 1980, p. 232):

$P_{\mathrm{g}}=\frac{n_{\mathrm{p}} k T}{\left(1-b n_{\mathrm{p}}\right)}$

where $b n_{\mathrm{p}}$ (with $n_{\mathrm{p}}$ being the numerical density of particles) is proportional to the ratio of the volume occupied by extended particles to the total available volume,

$b n_{\mathrm{p}}=a \frac{4 \pi}{3} \sum_{j} r_{j}^{3} n_{j}$.

A virial expansion at second order of the EOS of a gas formed by hard-spheres results in $a=4$. However, the value of the constant $a$ must be chosen so as to give the best agreement with experiments or, otherwise, with the best model predictions. In our case, we have chosen the equation of state of Saumon \& Chabrier (EOS-SC) to perform comparisons with our gas model. EOS-SC is based on a careful study of inter-particle interactions for hydrogen and helium gases (see Saumon, Chabrier \& Van Horn 1995). As illustrated in Fig. 1 , a value $a=1$ reproduces the EOS-SC results at $\log \rho \lesssim-0.6$, which is sufficient for calculations of He WD model atmospheres in the studied range $\left(T_{\text {eff }} \geqslant 2500\right)$. Calculations displayed in Fig. 1 correspond to a hydrogen gas with temperatures between 1995 and $6310 \mathrm{~K}$. The ideal gas equation $(a=0)$ predicts high densities as 


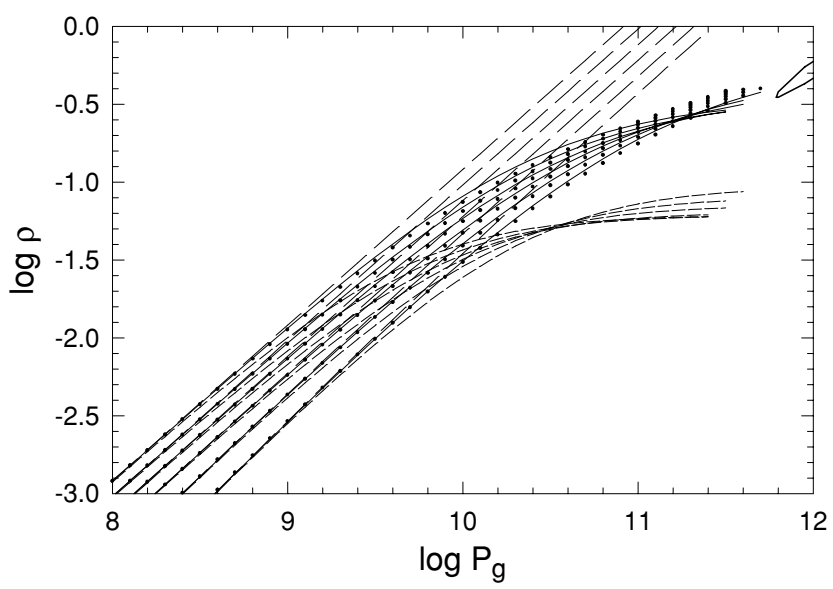

Figure 1. Comparison of density isotherms calculated from equation (10) with $a=0$ (long dashes), 1 (solid lines) and 4 (short dashes). Dots show the EOS-SC predictions. The temperature decreases from $\log T=3.8$ (right) to $\log T=3.3$ (left), with a constant spacing of $\Delta \log T=0.1$. The heavy line denote the plasma phase transition predicted by the EOS-SC (see text).

the pressure increases, whereas equation (10) with $a=4$ overestimates the excluded volume effect and yields too high a pressure for a given density. At low density all models recover the perfect gas limit. It is interesting to note in the figure the location of the plasma phase transition predicted by the EOS-SC, where the pressure ionization is a discontinuous process. The portion of the $\rho-P$ plane bounded by the heavy line corresponds to coexistent molecular and metallic hydrogen states. In the right-hand boundary curve, starting from the critical point $\left(\log P_{\mathrm{g}}, \log \rho\right)=(11.788,-0.456)$, lies the phase dominated by $\mathrm{H}_{2}$, whereas the left-hand boundary curve corresponds to the partially-ionized phase dominated by fluid metallic hydrogen.

In connection with our implementation of a non-ideal gas model for He WD atmosphere calculations, some words are in order. Saumon \& Chabrier (1991) found that nonlinear contributions to the configuration free energy (based on fluid perturbation theory) are necessary to yield the pressure dissociation of $\mathrm{H}_{2}$ molecules, such as is expected in hydrogen gas at high density $\left(\rho \approx 0.5 \mathrm{~g} \mathrm{~cm}^{-3}\right)$. They also show that a simple excluded volume interaction (such as that used in our gas model) cannot lead to pressure ionization. This result is confirmed by our calculations, which are based on the non-ideal gas model detailed in the present subsection. We display in Fig. 2 the fractional abundance of atomic hydrogen along isotherms calculated with different gas models. Results based on an ideal gas, our rigid-sphere gas model, and the EOS-SC, are represented by dotted lines, dashed lines and open circles, respectively. As density grows above $\log \rho \approx-2$, we found that a non-ideal contribution to Helmholtz energy based only on the excluded volume interaction increases the molecular formation with respect to ideal gas model calculations (using the classical Saha relation). In similar conditions, nevertheless, the atomic abundance along isotherms computed with EOS-SC becomes relatively shallow before reaching the pressure ionization-dissociation regime. Therefore, it seems essential to include nonlinear contributions in the configuration energy (besides the occupation probability formalism) of the gas for a proper treatment of pressure dissociation. However, we found it possible to represent with our model the gas behaviour predicted by the EOS-SC, adjusting the effective radius of molecules $\mathrm{H}_{2}$ (instead of implementing a more sophisticated gas model). In order to

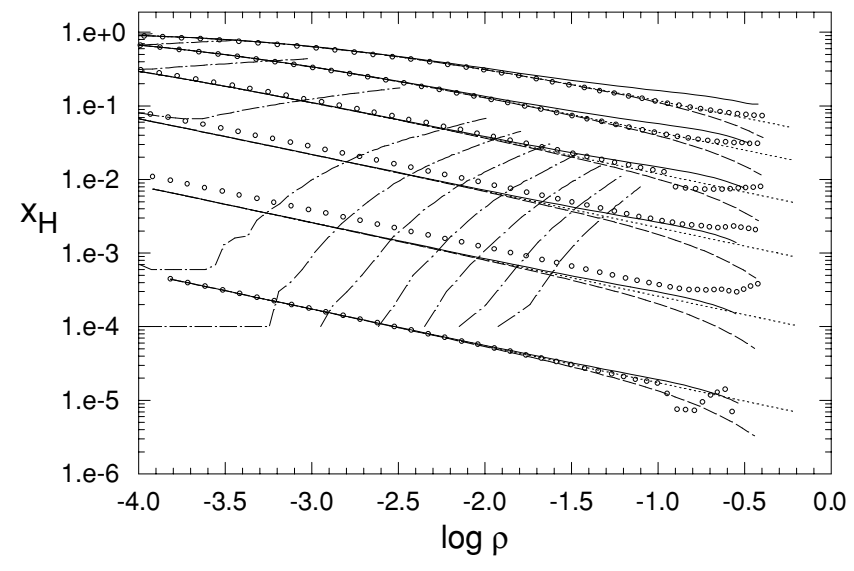

Figure 2. Concentration of atomic hydrogen along isotherms (from bottom to top) $\log T=3.3,3.4,3.5,3.6,3.7$ and 3.8. Dotted lines, dashed lines and circles represent calculations using the ideal gas model (with a density-dependent cutoff in the partition function of $\mathrm{H}$ atoms), occupational probability formalism and EOS-SC, respectively. Solid lines are calculated with the occupation formalism using the $\mathrm{H}_{2}$ effective radius increased to 1.4 of the original value given by equation (6). Dash-dotted lines represent atmosphere models at $\log g=8$ with $T_{\text {eff }}=2500$ (250) $4000(500) 6000$ (from right to left).

obtain a chemical equilibrium at high density in better agreement with EOS-SC calculations, we found it appropriate to use an $\mathrm{H}_{2}$ effective radius increased to 1.4 of its original (temperature- and density-dependent) value given by equation (6). ${ }^{1}$ This modification allows us to obtain more appropriate chemical abundances (solid lines in Fig. 2) in the regime of hight densities characteristic of deep layers of cool WD model atmospheres (dash-dotted lines), without altering the gas model predictions for lower densities.

\subsection{Opacity laws for a non-ideal gas}

It is observed in DA WD spectra (i.e. spectra showing hydrogen lines) that the high Balmer lines strongly overlap on the red side of the Balmer jump, forming a pseudo-continuum. This occurs similarly in other spectral series and it gives rise to a rather smooth curve for the opacity law instead of abrupt jumps in the series limits.

In the model atmospheres, the evaluation of the opacity at the line-merging region is often based on a cutoff of high-lying atomic levels and a shifting of the continuum edge down to the last line rising in the atomic model. According to Däppen et al. (1987, hereafter DAM), the occupational formalism of Hummer \& Mihalas offers an alternative approximation to compute pseudo-continuum opacities.

Imagine two atomic states, one lower energy level $i$ and another upper energy level $j$ with occupation probabilities $w_{i}$ and $w_{j}$, respectively. Based on the probabilistic interpretation of the factors $w$, DAM consider that a fraction of $w_{j}$ of the atoms have the bound state $j$ unperturbed, and the complementary fraction $1-w_{j}$ have the level $j$ 'dissolved', i.e. an atom in this latter fraction finds itself unbound and can be treated as a free level. The first class of states are simply called ' $j$ bound' and the second ones are referred as ' $j$ dissolved'. From this classification, DAM derive that atomic transitions $i \rightarrow j$ have a probability $w_{j} / w_{i}$ to be bound-bound transitions

\footnotetext{
${ }^{1}$ The new values of $r_{\mathrm{H}_{2}}(1.1-1.5 \AA)$ applied in our model fall within the dispersion of values found by different researchers, see Section 3.1.
} 
Table 1. Simulations for the atomic transition rate $i \rightarrow j$.

\begin{tabular}{llc}
\hline Model & \multicolumn{1}{c}{$j$ bound } & \multicolumn{1}{c}{$j$ dissolved } \\
\hline Mihalas (1966) & $w_{i} w_{j} n_{i} \Lambda_{i j}$ & $w_{i}\left(1-w_{j}\right) n_{i} \Lambda_{i j}$ \\
Däppen et al. (1987) & $\left(w_{j} / w_{i}\right) n_{i} \Lambda_{i j}$ & $\left(1-w_{j} / w_{i}\right) n_{i} \Lambda_{i j}$ \\
Hubeny et al. (1994) & $w_{j} n_{i} \Lambda_{i j}$ & $\left(1-w_{j}\right) n_{i} \Lambda_{i j}$ \\
Present interpretation & $w_{j} n_{i} \Lambda_{i j}$ & - \\
\hline
\end{tabular}

and a probability $1-w_{j} / w_{i}$ to be bound-free transitions (contributing to the pseudo-continuum opacity). Hubeny et al. (1994, hereafter HHL) proposed a generalization of the optical simulation of DAM to non-LTE conditions, but conserving the qualitative aspects of the original picture. Transition rates derived from these simulations are summarized in Table 1, together with an early proposal due to Mihalas (1966). We denote $\Lambda_{i j}$ as the transition probability per second from $i$ to $j$ corresponding to an isolated atom.

We note, however, that the optical simulations of DAM and HHL are not completely self-consistent with the Hummer-Mihalas formalism because the dissolved states have been incorporated in the theory a posteriori, when the stoichiometric equations were just established for particles species having unperturbed eigenenergies. Thus, for example, if (as permitted in DAM and HHL simulations) a fraction of atoms in a certain level $i$ can undergo transitions to the bound state $j$ and a complementary fraction of atoms in $i$ can be ionized at the same energy (dissolved state $j$ ), then the level $i$ does not have a unique energy value (that of an isolated atom) with respect to the continuum as assumed in the HummerMihalas framework. Indeed, in these simulations, the population equilibrium is calculated with ionization potentials without plasmainduced shifts, but simultaneously a certain fraction of atoms is permitted to be left in dissolved states (i.e. lies in the continuum) in transitions involving energies lower than the ionization energies. This inconsistency suggests a revision of the opacity theory.

Our interpretation of the gas optical properties in the HummerMihalas formalism is as follows. First, the dissolved states are not possible in the gas model, because its existence implies a violation of the particle distribution as dictated by the stoichiometric equations of ionization used. Then, the classification of atoms according to state $j$ bound or dissolved is illegitimate for the present state of the theory. Secondly, the factors $w_{i}$ must participate in the atomic transition rates, since they decide the availability of the atomic states, as is clearly expressed by equation (1).

Thus, the rate of transition $i \rightarrow j$ must be zero when $w_{j}=0$ (i.e. when $j$ is an unavailable state) and equal to the standard rate $n_{i} \Lambda_{i j}$ when $w_{j}=1$ (atom not perturbed). Intermediate-rate values should be found when $0<w_{j}<1$. Therefore, the simplest heuristic representation of the transition rate for a non-ideal gas (characterized by the Hummer-Mihalas formalism) is

$n_{i} \Lambda_{i j} w_{j}$.

We can interpret $\Lambda_{i j} w_{j}$ as the (conditional) probability that an atom in state $i$ makes a transition to state $j$ if this is available. ${ }^{2}$ The occupation probability $w_{i}$ of the initial state in the transition is not explicitly present in rate (12), because the availability of starting atoms for this process is just given by the present population

\footnotetext{
${ }^{2}$ This conditional probability was also proposed by HHL (see Table 1 ), but as a result of the decomposition of the transition rate $n_{i} \Lambda_{i j}$ into two terms, one referred to $j$ as a bound state and the other term corresponded to $j$ being a dissolved state.
}

$n_{i}$ (this is a difference from the proposal of Mihalas 1966 - see Table 1).

Despite our critique of the current optical simulations based on the occupational formalism, we considered that the proposals of DAM and HHL are useful (state-of-the-art) approximations for the representation of the opacity in the line-merging region, at least for Balmer, Paschen and other series (except for the Lyman series see below). In fact, for interpreting current high-accuracy spectrophotometric observations, models including pseudo-continuum opacity are necessary. In this sense, the phenomenological theory introduced by DAM provides an approximation to the solution. In the absence of a self-consistent theory, for the opacity laws applied to our calculations we take from the DAM proposal the contribution to the pseudo-continuum cross-section as we now explain.

In our model atmospheres, the total opacity coefficient (corrected for stimulated emission) containing all contributions of level $i$ is calculated as

$\chi_{i j, v}=n_{i}\left[\sum_{j} \alpha_{i j, v} w_{j}+D_{i, v} \alpha_{i k, v} w_{k}\right]\left(1-e^{-h v / k T}\right)$.

The first term in the square brackets corresponds to the boundbound opacities (with cross-section $\alpha_{i j, v}$ ) following the prescription (12). The second term in equation (13) represents the boundfree absorption in the same prescription (i.e. with the corresponding cross-section $\alpha_{i k, v}$ multiplied by the occupation probability of the final state; for example, in the case of $\mathrm{H}$ atoms, $w_{k}=1$ ) modified by a factor $D_{i, v}$ that takes into account the pseudo-continuum contribution.

The so-called dissolved fraction $D_{i, v}$ extends the photoionization cross-section to frequencies below the frequency threshold corresponding to an isolated atom. DAM give for $D_{i, v}$ the expression

$D_{i, v}=\frac{w_{i}-w_{n^{*}}}{w_{i}}$

where $w_{n^{*}}$ is the occupation probability calculated for a fictitious (unless $n^{*}$ is integer) state with effective quantum number $n^{*}$ given by

$n^{*}=\left(\frac{1}{n_{i}^{2}}-\frac{h v}{\chi}\right)^{-1 / 2}$

where $\chi$ is the ionization potential of the species considered. For imaginary $n^{*}$, we define $D_{i, v}=1$ and the usual bound-free opacity is recovered in equation (13).

Conceptually, the opacity law given in equation (13) is different from those proposed by DAM and HHL. However, in practice all these proposals give similar results because the most important spectral series (Lyman, Balmer and Paschen) have low-lying starting levels for which the occupation probabilities are very close to unity $\left(w_{i}=1\right)$.

Finally, it is worth noting that the absorption law (equation 13) computed with $D_{i, v}$ given by equation (14) yields an unphysical pseudo-continuum Lyman opacity for WD stars (Bergeron, Ruiz \& Leggett 1997). This theoretical opacity is so large that it becomes dominant over the total opacity, even in the infrared. A modified dilution factor $D_{i, v}$ for Lyman opacity was considered by Bergeron (2001) in order to fit the spectrum of the halo WD 0346 + 246. However, this ad hoc proposal is based on arbitrary physical assumptions and needs further investigation. We have preferred not to include this 
source of opacity in our calculations until a more appropriate theory becomes available.

\subsection{Hydrogen-line opacity}

Line opacities of the Lyman, Balmer and Paschen series have been included in the present model-atmosphere calculations. However, in this work we are not interested in reproducing detailed observed line profiles but instead in computing the radiated energy distribution including line blanketing. So, theoretical line opacities have been incorporated in the model-atmosphere calculation in an approximate way.

Because of the high densities encountered in WD atmospheres, the pressure broadening is usually the dominant line-broadening mechanism and the profiles $\left(\sigma_{v}\right)$ can be assumed Lorentzian:

$\sigma_{v}=\frac{\gamma}{\pi\left[\left(v-v_{o}\right)^{2}+\gamma^{2}\right]}$,

where $v_{o}$ is the central frequency of the line. The total damping width $\gamma$ is the sum of the natural, linear Stark and van der Waals damping widths (in $\mathrm{rad} \mathrm{s}^{-1}$ ),

$\gamma=\left(\gamma_{\text {nat }}+\gamma_{\text {Stark }} / c+\gamma_{\mathrm{vdW}}\right) / 4 \pi$.

The broadening effects have been considered with damping parameters given by Anderson (1989). The linear Stark width is a fit to results of Griem (1960), and the van der Waals width is a fit to the $d-f$ transitions of Derrider \& Van Rensbergen (1976). Taking into account the equivalent widths typically observed for WDs, we found it necessary to include an additional parameter $c$ to reduce the Stark width given by the Anderson's formula (specially in hot stars, $T_{\text {eff }}>10000 \mathrm{~K}$, where the gas is ionized and Stark broadening dominates over the other broadening mechanisms). For that purpose, we empirically found it appropriate to set $c$ equal to 3 . Fig. 3 shows a comparison of the equivalent widths of the $\mathrm{H} \alpha$ and $\mathrm{H} \beta$ lines observed for a group of WDs (from Greenstein 1986; Bergeron,

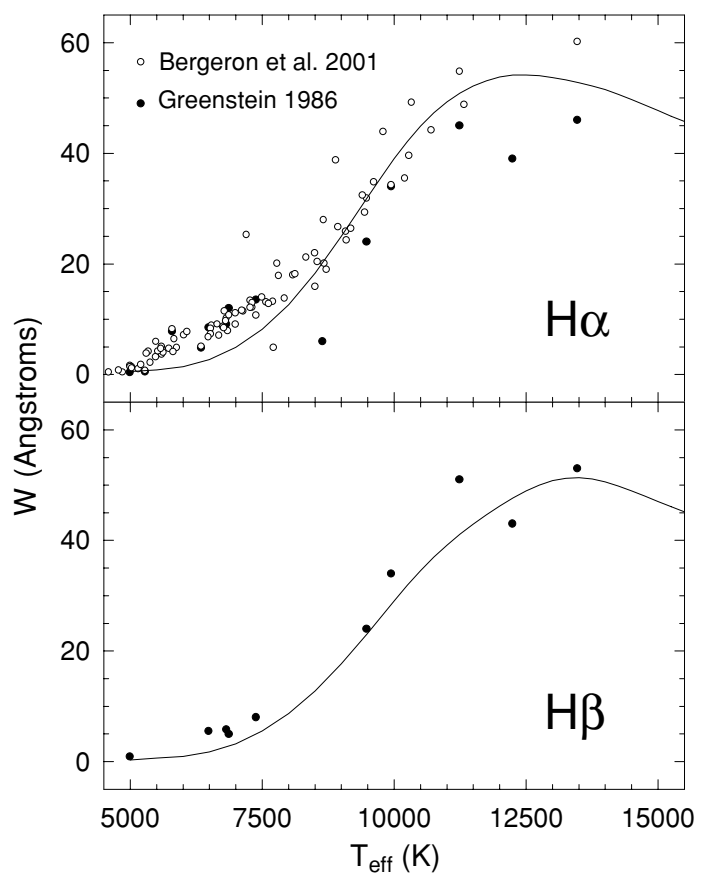

Figure 3. Equivalent widths of $\mathrm{H} \alpha$ and $\mathrm{H} \beta$ for a group of WD stars, as a function of $T_{\text {eff. }}$. Solid lines indicate the predictions of our $\log g=8$ models.
Leggett \& Ruiz 2001) with those calculated from our model atmospheres.

Since the radiative flux and atmosphere structure is particularly sensitive to the profile of $\mathrm{L} \alpha$, a more accurate theory of line broadening was used for this line. To this end, we employed for $L \alpha$ the Stark-broadening tables of Vidal, Cooper \& Smith (1973).

In hot model atmospheres (e.g. $T_{\text {eff }} \approx 15000 \mathrm{~K}$ ), the Lyman lines can 'block' up to 50 per cent of the energy transported in the continuum spectrum. Because its strong influence over the structure of the atmosphere, Lyman line opacity has been included in the energy-balance and transfer equations during the iterative temperature correction procedure to compute each model atmosphere (see Rohrmann 2001). Then, from the thermodynamic stratification of the converged model, we computed the emergent flux including also formation of Balmer and Paschen line series, the effect of which on the temperature-pressure structure was considered negligible.

We chose about six-hundred frequency points to represent the radiation field, as required to calculate in detail the emission-opacity coefficients from all continuum and line processes included in the atmosphere code.

\subsection{Collision-induced absorptions}

In mixed hydrogen and helium gases, colliding pairs of atoms and molecules such as $\mathrm{H}_{2}-\mathrm{H}_{2}, \mathrm{H}_{2}-\mathrm{He}$ and $\mathrm{H}-\mathrm{He}$ can be sources of CIA opacity. Recent calculations of $\mathrm{H}_{2}-\mathrm{He}$ absorption coefficients are available from Jorgensen et al. (2000) and cover temperatures between 1000 and $7000 \mathrm{~K}$ for the $25-20088 \mathrm{~cm}^{-1}$ frequency region. Also, most up-to-date CIA opacities due to $\mathrm{H}_{2}-\mathrm{H}_{2}$ have been calculated by Borysow, Jorgensen \& Fu (2001) for $1000 \leqslant T \leqslant$ $7000 \mathrm{~K}$ and frequencies in the range $20-20000 \mathrm{~cm}^{-1}$. Furthermore, $\mathrm{H}-\mathrm{He}$ CIA data based on recent quantum mechanical computations by Gustafsson \& Frommhold (2001) are now available for temperatures in the range $1500-10000 \mathrm{~K}$ and frequencies in the range 50 $11000 \mathrm{~cm}^{-1}$.

All these CIA opacities were included in our model calculations. Extrapolation (in logarithmic scales) to temperatures and frequencies beyond the data limits, when necessary, were used in our models. Fig. 4 illustrates the CIA values calculated by Borysow and co-workers (solid lines) and our corresponding fits (dotted lines).

\subsection{Photometric calibration of models}

In order to compute colour indices in the UBVRI-JHKL photometry system, a set of zero points have been calculated. These calibration constants are necessary to convert the theoretical model fluxes $\left(F_{X}, F_{Y}\right)$ (integrated over filter response functions) to observational colours $X-Y$ (corresponding to certain filters $X$ and $Y$ ),

$X-Y=-2.5 \log \left(F_{X} / F_{Y}\right)+C_{X-Y}$.

The zero points $C_{X-Y}$ were set by requiring that the flux of the star Vega has zero colours. We employed the synthetic flux of the model ( $T_{\text {eff }}=9400 \mathrm{~K}, \log g=3.95$ ) calculated by Kurucz (1979) for Vega, and the observed fluxes of Vega measured by Hayes (1985). The zero points computed from these fluxes are showed in Table 2, together with the constants given by Bergeron et al. (1997), which we used in previous calculations (Rohrmann 2001; Serenelli et al. 2001). For the present calculations of the broadband colour indices and for reasons of internal consistency, we used the zero point derived from the Vega model provided by Kurucz, because this flux covers all broadband regions. 


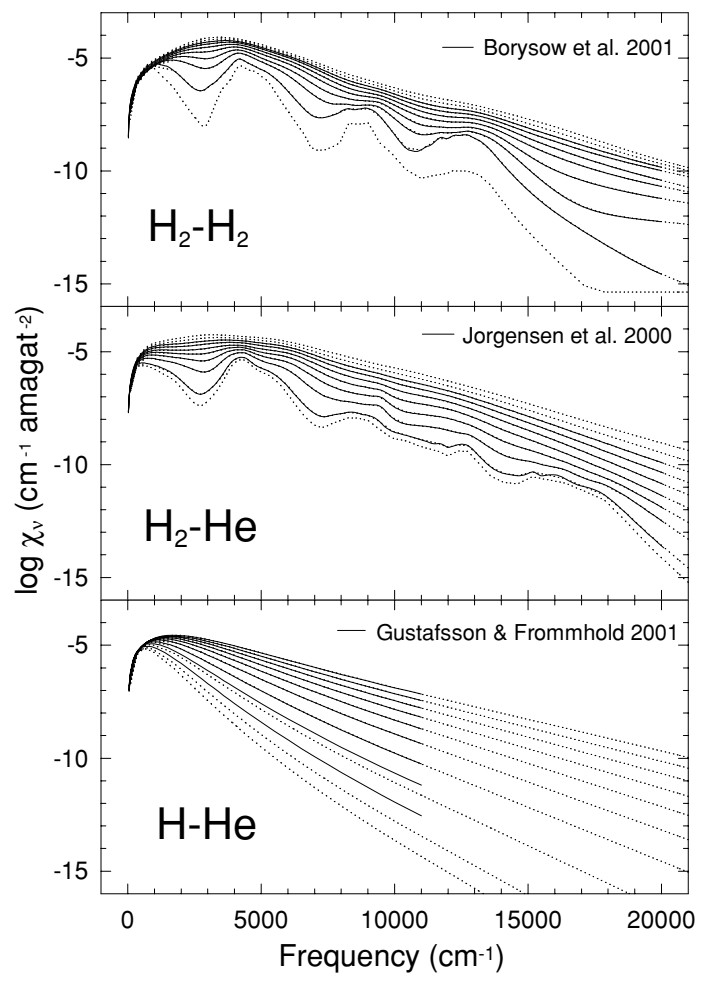

Figure 4. Collision-induced opacity corresponding to $\mathrm{H}_{2}-\mathrm{H}_{2}, \mathrm{H}_{2}-\mathrm{He}$ and $\mathrm{H}-$ He derived by Borysow et al. (2001), Jorgensen et al. (2000) and Gustafsson \& Frommhold (2001), respectively. Dotted lines indicate our calculations based on those results, for 500, 1000 (1000) $9000 \mathrm{~K}$ from bottom to top. Solid lines show calculations of Borysow et al. and Jorgensen et al. for temperatures $T=1000(1000) 7000 \mathrm{~K}$, and those of Gustafsson \& Frommhold for $T=1500,2250,3000$ (1000) $9000 \mathrm{~K}$ (all opacities grow with increasing temperature).

\section{RESULTS}

We have used the sets of cooling sequences of Serenelli et al. (2001) to determine accurate spectra and colour indices for He WD models with stellar masses 0.406, 0.360, 0.327, 0.292, 0.242, 0.196 and $0.169 \mathrm{M}_{\odot}$. These models were obtained using the WD evolutionary code described in Althaus et al. (2001) in a self-consistent way with the predictions of detailed non-grey model atmospheres (Rohrmann 2001), element diffusion and the history of the WD progenitor. More details of our current model grid can be found in the cited literature and references therein. Additionally, two new evolutionary sequences corresponding to 0.160 and $0.148 \mathrm{M}_{\odot}$ have been calculated for a proper interpretation of recent observational data.

Table 2. Zero points $C_{X-Y}$ computed using (a) a synthetic spectrum from Kurucz (1979) and (b) the observed fluxes of Vega measured by Hayes (1985), and $(c)$ those provided by Bergeron et al. (1997).

\begin{tabular}{lccc}
\hline Colour index & $(a)$ & $(b)$ & $(c)$ \\
\hline$U-B$ & -0.471 & -0.499 & - \\
$B-V$ & +0.596 & +0.625 & +0.609 \\
$V-R$ & +0.555 & +0.560 & +0.557 \\
$V-K$ & +4.839 & - & +4.886 \\
$R-I$ & +0.703 & +0.700 & +0.704 \\
$J-H$ & +1.099 & - & +1.082 \\
$H-K$ & +1.093 & - & +1.135 \\
$K-L$ & +1.854 & - & - \\
\hline
\end{tabular}

For the present work, particular attention has been paid to the calculation of the synthetic spectra with an improved version of the atmosphere code, which treats hydrogen-line blanketing and nonideal effects in the gas within the occupation formalism of Hummer \& Mihalas (1988), as described in Section 2.

\subsection{Consequences of the new input physics}

We first analyse the implications of using a non-ideal gas model for calculating model atmospheres of He WDs. The consequences of non-ideal effects in the equation of state for WD atmospheres have been explored previously in the context of hydrogen atmospheres (Bergeron et al. 1991; Saumon \& Jacobson 1999), pure helium models (Böhm et al. 1977; Kapranidis 1983; Bergeron, Saumon \& Wesemael 1995) and models with mixed composition (Bergeron et al. 1995; Bergeron 2001). Therefore, we only refer here to those results particularly significant for the present study of He WDs.

We found that the effects of a non-ideal gas are manifold. The interaction amongst the particles determines through the partition functions the chemical equilibrium of the gas, which can affect its thermodynamic behaviour (equations of state and response functions: adiabatic gradient, specific heats) and opacity properties (changes operated through variations of the abundances of absorbers and formation of pseudo-continuum spectra). These modifications can yield changes over the hydrostatic stratification of the atmosphere and emergent energy spectrum of the He WD stars in two regimes: in cool models characterized by molecular formation and in hot models where the hydrogen line opacity is relevant. We can appreciate the consequences of this new input physics in the colourcolour diagrams displayed in Fig. 5, where our current and old calculations (Rohrmann 2001) are compared with those obtained from other atmosphere codes (data kindly provided by Bergeron and Saumon).

In cool atmospheres $\left(T_{\text {eff }} \lesssim 4000 \mathrm{~K}\right)$, which are composed of a dense fluid formed mainly by $\mathrm{H}_{2}$ molecules, the main effects of nonideal gas have their origin in a severe reduction of the occupation probabilities of particle states, even ground states. This is illustrated in Fig. 6, where we note how low-excitation states of $\mathrm{H}$ and $\mathrm{H}_{2}$ are perturbed with increasing pressure in the surface of a $0.292-\mathrm{M} \odot$ He WD at $T_{\text {eff }}=2500 \mathrm{~K}$. Because of the low temperatures present in these atmospheres, the charged particle density is low and the main contribution to the occupation probabilities comes from the excluded volume interaction. Molecules have effective radii something higher than the radius of the ground state of the $\mathrm{H}$ atom and, therefore, they experience perturbations with larger intensity. However, high-lying levels of the hydrogen atom are weakly bound and consequently easily destroyed, even in the outer layers of the atmosphere.

It is not possible to predict if an increasing density favours the dissociation or formation of molecules, because this outcome (the chemical equilibrium) strongly depends on the radii assigned to atoms and molecules. With the current input physics (involving the occupation formalism of Hummer \& Mihalas), we found that the non-ideal effects increase the $\mathrm{H}_{2}$ dissociation into $\mathrm{H}$ atoms in the atmospheric region where the continuum radiation is formed and particularly towards deep layers in cool atmospheres (see Fig.2). As a consequence, the importance of the CIA opacity on the radiative transfer is reduced as compared with a model based on ideal gas. The resulting emergent flux is less displaced to shorter wavelengths with respect to our early calculations based on an ideal gas (Rohrmann 2001). Fig. 5 shows for $T_{\text {eff }} \leqslant 3000 \mathrm{~K}$ some redder colours (especially $R-I$ and $V-K$ ) resulting from models with 

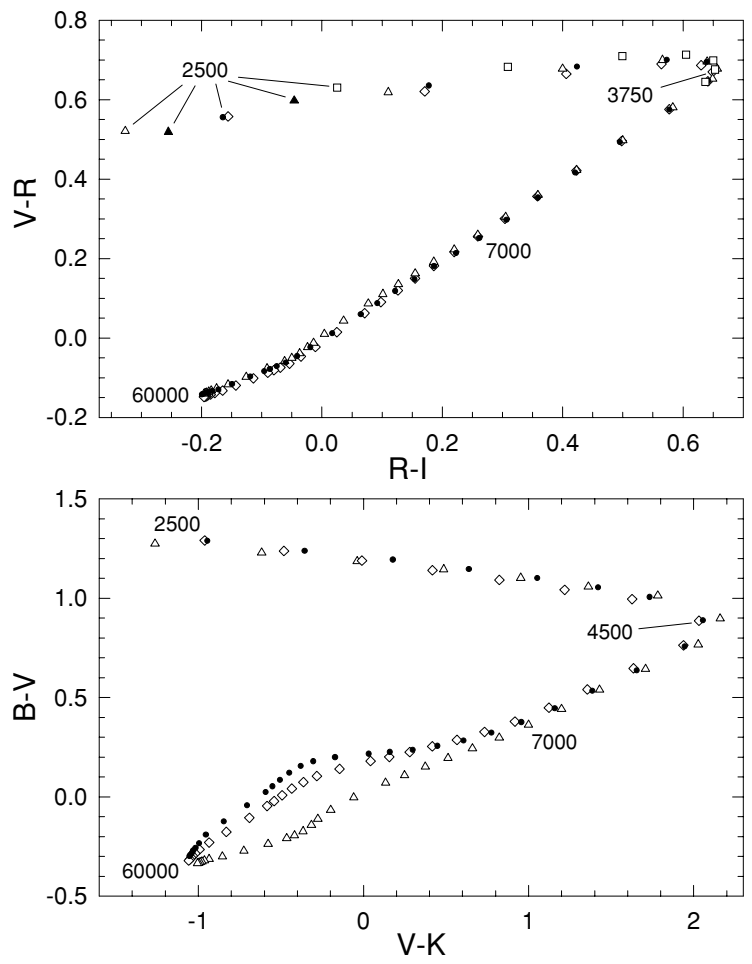

Figure 5. Colour-colour diagrams for $\log g=8$ models with $T_{\text {eff }}=$ 2500 (250), 4000 (500), 10000 (1000), 17000,20000 (5000) and $60000 \mathrm{~K}$. Open diamonds denote our current calculations, whereas filled circles and open squares correspond to Bergeron (private communication), and Saumon \& Jacobson $\left(1999, T_{\text {eff }} \leqslant 4000\right)$, models, respectively. Our old results based on ideal gas theory and without hydrogen-line and pseudo-continuum opacities are indicated by open triangles. Some $T_{\text {eff }}$ values are labelled on the plots. The $(V-R, R-I)$ diagram shows also results (filled triangles) for the $T_{\text {eff }}=2500$ model based on non-ideal gas with different values of the effective radius of $\mathrm{H}_{2}$ molecules (see text).

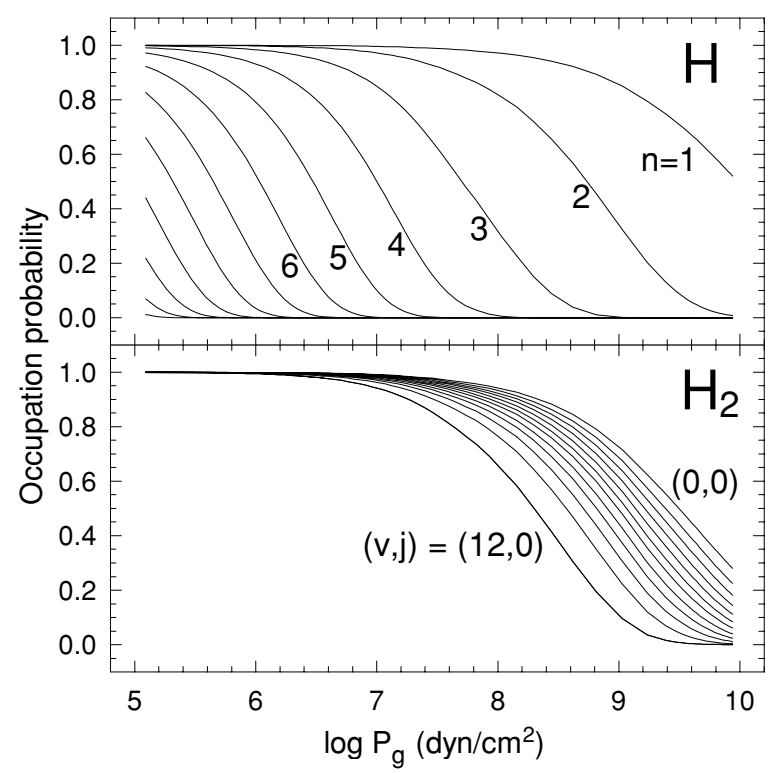

Figure 6. Occupation probability of lower levels of $\mathrm{H}$ and lower vibrational states $v$ of $\mathrm{H}_{2}(0 \leqslant v \leqslant 12$, with rotational quantum number $j=0)$ as a function of the gas pressure, for a $0.292-\mathrm{M}_{\odot} \mathrm{He}$ WD model with $T_{\text {eff }}=$ $2500 \mathrm{~K}$. non-ideal gas (open diamonds) compared with those obtained using ideal gas theory (open triangles). This behaviour is in qualitative agreement with results reported by Saumon \& Jacobson (1999) for hydrogen-model atmospheres. Their $\log g=8$ sequence starting at $T_{\text {eff }}=4000 \mathrm{~K}$ (reproduced on the $V-R$ versus $R-I$ diagram in Fig. 5) appears somewhat redder with respect to our colour computations, which matches the sequence of Bergeron very well. Good agreement is also obtained between our models and those of Bergeron for the sequence in the $(B-V, V-K)$ diagram.

However, we note that the effective radius assigned to molecules can strongly affect the spectrum of very cool models. For example, if the radius of $\mathrm{H}_{2}$ is reduced by 10 per cent of the current value used in our model, the formation of molecules is favoured, thus increasing the CIA opacity and pushing the emergent flux toward higher frequencies. The contrary effect follows when the radius of $\mathrm{H}_{2}$ is increased by 10 per cent of the original value. As a consequence, these exploratory modifications yield, for example, changes of about $0.1 \mathrm{mag}$ in $R-I$ for cool WDs (filled triangles in Fig. 5). Therefore, we conclude that a reliable description of the spectrum of very cool WDs rests on a satisfactory representation of molecular interactions. However, the investigation of properties of dense molecular hydrogen gas often yields different results according to the techniques and methods used (e.g. Saumon et al. 1995). Thus, for example, a variety of effective radii have been considered for $\mathrm{H}_{2}$ molecules from different studies, e.g. $r_{\mathrm{H}_{2}}=1.48$ (Graboske et al. 1969), 0.73-0.82 (Ross, Ree \& Young 1983), 1.23 (Robnik \& Kundt 1983), 1.45 (Mihalas, Däppen \& Hummer 1988), 0.81-1.10 ̊̊ (Saumon \& Chabrier 1991). Additional progress in the study of dense gases could prove useful in determining more accurate spectra for very cool WDs.

For hot He WD atmosphere models $\left(T_{\text {eff }} \gtrsim 8000 \mathrm{~K}\right)$, the lower atomic levels remain almost unperturbed, so that the chemical equilibrium is not seriously affected by non-ideal effects. However, perturbations amongst neighbouring particles tend to destroy the high energy levels and affect the line series limits by removing higher series members. In addition, the continuum opacity advances over the regions where the lines overlap forming a pseudo-continuum. This behaviour is described by the occupational formalism together with the optical simulation synthesized in equation (13).

We analyse in Fig. 7 the importance of the non-ideal effects in the gas on the Balmer line spectrum. Two spectra have been

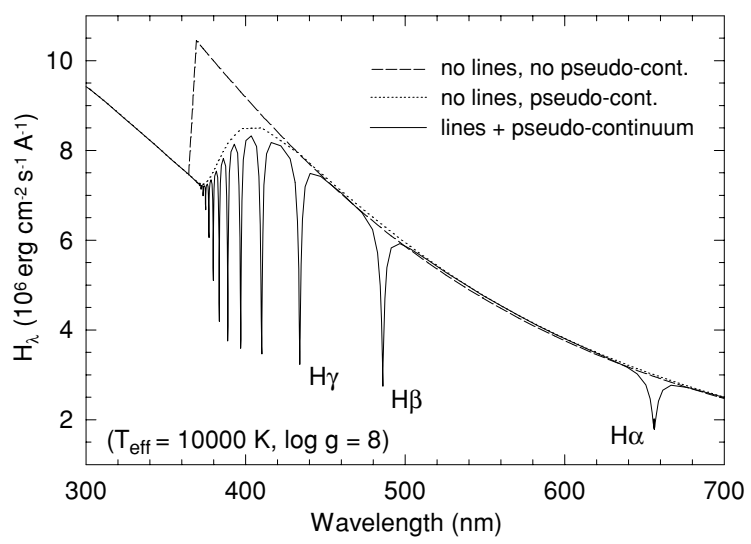

Figure 7. Emergent radiative energy from a hot H-model atmosphere in the Balmer line region using different opacity laws. The pseudo-continuum opacity simulated with the DAM theory produces a shift of the Balmer discontinuity towards the red and changes the shape of the continuum spectrum. 
Table 3. Main properties of the atmosphere codes whose results are compared in Fig. 5. Notation is as follow. DAM: optical simulation of Däppen et al. (1987); HM: occupation formalism of Hummer \& Mihalas (1988); NDHM: HM occupation formalism including plasma correlation effects from Nayfonov et al. (1999); SCVH-EOS*: equation of state (treating $\mathrm{H}_{2}, \mathrm{H}, \mathrm{H}^{+}$and $\mathrm{e}^{-}$) based on the model of Saumon et al. (1995) and modified for reproducing available high-pressure data (Saumon et al. 2000).

\begin{tabular}{lcccccr}
\hline Model atmosphere & $(P, T, \rho)$ relation & Chemical equilibrium & $r_{\mathrm{H}}(\AA)$ & $r_{\mathrm{H}_{2}}(\AA)$ & Opacity law & $\mathrm{H}_{2}-\mathrm{H}_{2}$ absorption \\
\hline Bergeron & Ideal gas & $\mathrm{HM}$ & $0.50^{a}$ & $-{ }^{b}$ & DAM & Borysow et al. 1997 \\
Saumon \& Jacobson & SCVH-EOS* & SCVH-EOS* $+\mathrm{HM}^{c}$ & 0.65 & 1.07 & Standard & Borysow et al. 1997 \\
Present work & Equation (10) & NDHM & $0.58-0.86$ & $1.1-1.5$ & Equation (13) & Borysow et al. (2001) \\
\hline
\end{tabular}

${ }^{a}$ Value adopted in order to reproduce observed profiles of higher Balmer lines (Bergeron et al. 1991).

${ }^{b}$ The $\mathrm{H}_{2}$ population was not computed in the $\mathrm{HM}$ formalism but with a standard Saha-like formula.

${ }^{c}$ Species $\mathrm{H}^{-}, \mathrm{H}_{2}^{+}, \mathrm{H}_{3}^{+}$are treated as perturbative species in the equilibrium using HM formalism (Lenzuni \& Saumon 1992).

calculated by neglecting the line opacities in order to illustrate the importance of the pseudo-continuum opacity. For an ideal gas model without line absorption, the energy distribution shows an abrupt Balmer discontinuity (the dashed line in the figure). The inclusion of a pseudo-continuum opacity based on the DAM optical simulation extends the bound-free absorption toward longer wavelengths, yielding a marked change in the continuum spectrum (the dotted line). This behaviour results from the ionization of perturbed $\mathrm{H}$ atoms to states with energy lower than that corresponding to the isolated atom model. When the line opacity is considered (the solid line in the figure), we note that the spectral region where the lines overlap is completely dominated by the pseudo-continuum opacity. Accordingly, the intensity of higher series members is diminished as a result of the low occupation probabilities of final states in the bound-bound transitions.

The colour calculations, particularly those involving magnitudes from broadbands lying over the Balmer jump, are sensitive to the inclusion of hydrogen-line blanketing and pseudo-continuum process, as can be observed in Fig. 5. Line opacity and Stark ionization strongly contribute to the absorption in ultraviolet and visible spectra, and significantly affect the colours for $T_{\text {eff }} \gtrsim 8000 \mathrm{~K}$, mainly through the $B$ photometric bandpass. Our new calculations agree reasonably well with those of Bergeron. The remnant discrepancies amongst colour calculations from the different atmosphere codes shown in Fig. 5 could be due to the CIA opacity used for the cool end of the model sequence (we use recently available data) and, presumably, to the constitutive physics concerning the non-ideal effects used in the codes. Table 3 summarizes the properties of the atmosphere codes, the results of which are compared here.

Some representative emergent spectra obtained from our new calculations can be appreciated in the cooling sequence of the 0.292- $\mathrm{M}_{\odot}$ He WD model displayed in Fig. 8. These models correspond to $T_{\text {eff }}=15000,10000,7000,2500 \mathrm{~K}$, and $\log g=7.092$, $7.195,7.267,7.384$, respectively, and have a pure hydrogen atmosphere. Several changes can be appreciated in Fig. 8 comparing the new spectra (the solid lines) with our previous determinations (the dashed lines) obtained without line opacity and using ideal gas theory. In hot models, the Lyman line series forces the flux to emerge at lower frequencies; even the global change in the energy distribution of the relatively-cool $T_{\text {eff }}=7000 \mathrm{~K}$ model is affected by the Lyman line absorption (owing mainly to the red wing of $L \alpha$ ). As mentioned above, the variations in the spectrum of the cool model $\left(T_{\text {eff }}=\right.$ $2500 \mathrm{~K})$ are due to both non-ideal effects and updated CIA opacity incorporated in the atmosphere code. The difference with the blackbody emission (dots) is a remarkable property of the advanced stages of cooling of He WD stars and WDs in general.
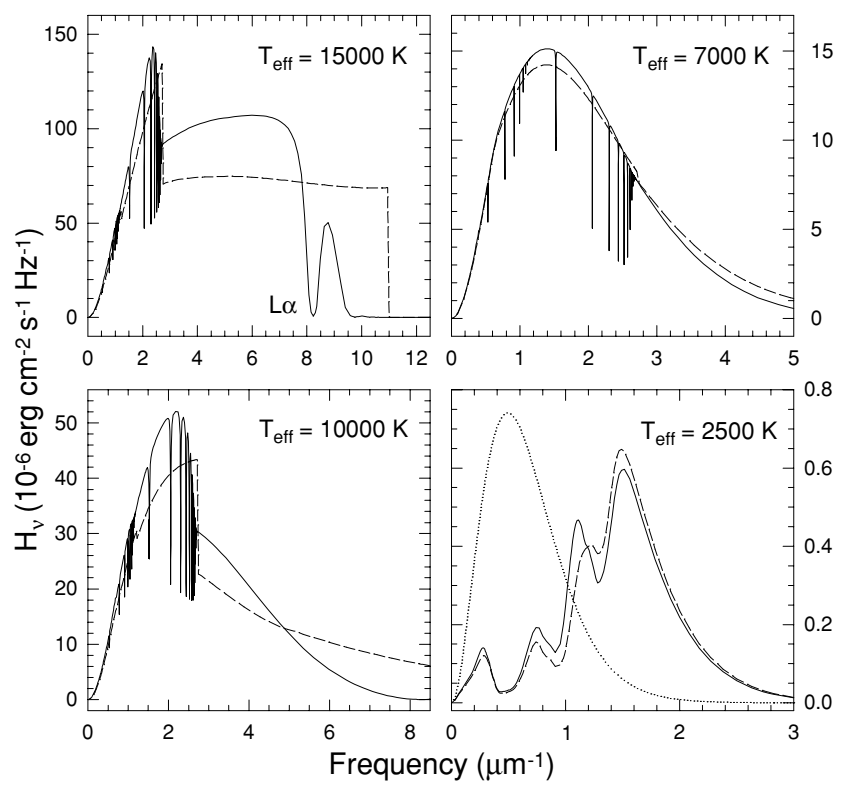

Figure 8. Spectra calculated for different values of $T_{\text {eff }}$ using the $0.292-\mathrm{M}_{\odot}$ He WD model. The solid lines indicate the present calculations including line opacity, non-ideal gas model and up-to-date CIA opacity, while the dashed lines represent our old calculations without these improvements. For $T_{\text {eff }}=2500$, the blackbody spectrum at the same temperature is also displayed (dots).

\subsection{Helium-core white dwarf photometry}

In Fig. 9 we show some computed colour-colour diagrams for our He WD models (solid lines), compared with WD observations (symbols). The cooling sequences start near the lower left corner and extend towards the upper right corner before showing a turn-off in some colours such as $V-I, V-K$ and $J-H$. The models cover the range $0.148-0.406 \mathrm{M}_{\odot}$. In the case of the lowest-mass models $\left(0.148,0.160\right.$ and $\left.0.169 \mathrm{M}_{\odot}\right)$, the evolution after the end of mass-loss episodes is slow, so they can age up to 1-1.5 Gyr before reaching the knee (highest $T_{\text {eff }}$ point) and starting to cool down. In view of this fact, there is a loop at the hot end of their sequences that can be appreciated in the curves in Fig. 9. We notice that our sequences fall along the bulk of the observations and expand towards cooler regions ( $T_{\text {eff }} \lesssim 3500 \mathrm{~K}$ ) of the diagrams, where there is little observational information about WD stars yet. The dotted lines in Fig. 9 represents our old computations for the $0.292 \mathrm{M}_{\odot}$ model (Serenelli et al. 2001). The differences between the new calculations and the previous approximations are significant for various 
Table 4. Selected stages for 0.148 - and $0.160-\mathrm{M}_{\odot}$ He WD models.

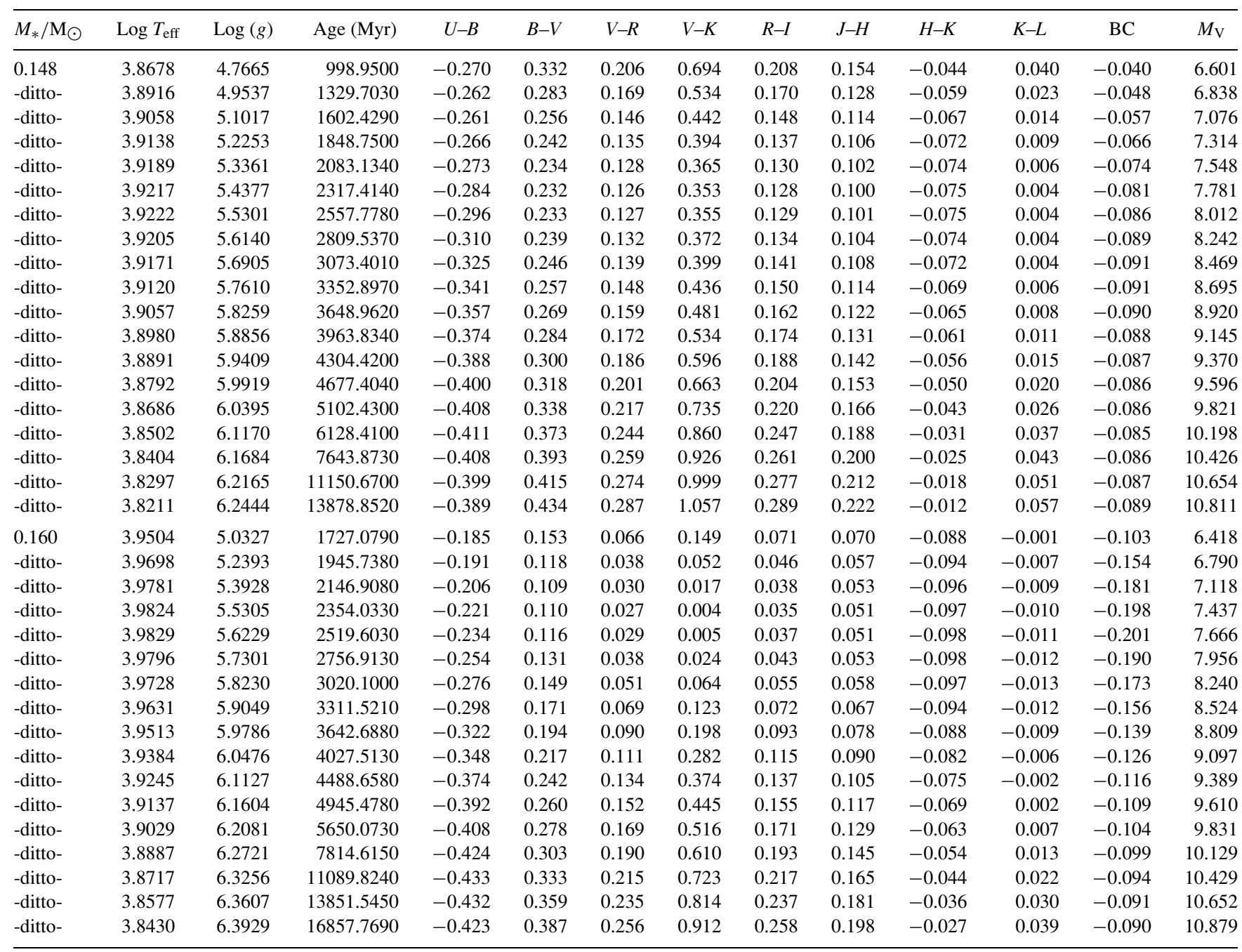

colours (affected by hydrogen-line opacity and non-ideal effects), particularly at high and very-low temperatures.

Figs 10 and 11 display absolute visual magnitude $M_{\mathrm{V}}$ versus $U-V$ and $V-I$ colours, respectively, for our He WD models covering $0.148-0.406 \mathrm{M}_{\odot}$. As already mentioned in Serenelli et al. (2001), He WD models with mass greater than $\approx 0.18 \mathrm{M}_{\odot}$ (in the case of progenitors of solar metallicity) can reach high magnitudes within cooling times less than $15 \mathrm{Gyr}$, owing to thermonuclear hydrogen shell flashes induced by element diffusion (Althaus et al. 2001). Collision-induced absorption by $\mathrm{H}_{2}$ causes turnover to bluer $V-I$ at $M_{\mathrm{V}} \approx 15.5-16.5$, depending on the stellar mass, whereas the $U-V$ index monotonically reddenes with decreasing $T_{\text {eff. Molecular opac- }}$ ity also yields a slow cooling rate in the later stages of the evolution. In the interests of comparison, the old computed tracks for 0.169 and $0.406 \mathrm{M}_{\odot}$ (dotted lines in the colour-magnitude diagrams) have been included in the figures. Note that the current improvements in the atmosphere code (especially the inclusion of hydrogen-line opacity) yield significant changes in the colour values at hot WD stages and, therefore, at low magnitudes. The $0.169-\mathrm{M}_{\odot}$ sequence now appears approximately 0.2 mag redder in the $U-V$ index, and 0.1 mag bluer in $V-I$ at early stages. The open circles in Fig. 10 correspond to a group of seemingly single WDs observed by Saffer et al. (1998), whereas open triangles and circles in Fig. 11 represent
DA and non-DA WDs, respectively, from Bergeron et al. (2001). It is interesting to note that our massive He WD sequences follow the red edge of the bulk of the observational samples. As reference, two ultracool WDs ( $T_{\text {eff }}$ below $4000 \mathrm{~K}$ ) are included in the figures (open squares), LHS 3250, characterized by a strong infrared flux deficiency (Harris et al. 1999), and WD 0346+246, which has an atmosphere of mixed hydrogen and helium composition (Bergeron 2001; Oppenheimer et al. 2001).

As stated in the introduction, very-low-mass He WDs have begun to be detected or inferred in globular clusters. Recently, Taylor et al. (2001) have presented observational evidence for He WD candidates in the cluster NGC 6397. Additionally, Edmonds et al. (2001) reported the optical detection of the He WD companion of mass $M \lesssim 0.17 \mathrm{M}_{\odot}$ to a millisecond pulsar in the globular cluster 47 Tucanae. In view of these considerations, we judge it to be worthwhile to extend the evolutionary calculations presented in Serenelli et al. (2001) to less-massive He WDs, thus enabling an appropriate comparison with the above-mentioned observations. To this end, we computed the evolution of He WDs with stellar masses of 0.148 and $0.160 \mathrm{M}_{\odot}$. Element diffusion, nuclear burning and the history of the WD progenitor are taken into account (see Serenelli et al. 2001, for details). Realistic initial models are obtained by abstracting mass from a $1 \mathrm{M}_{\odot}$ model. Because, in the case of very-low-mass He WDs, 

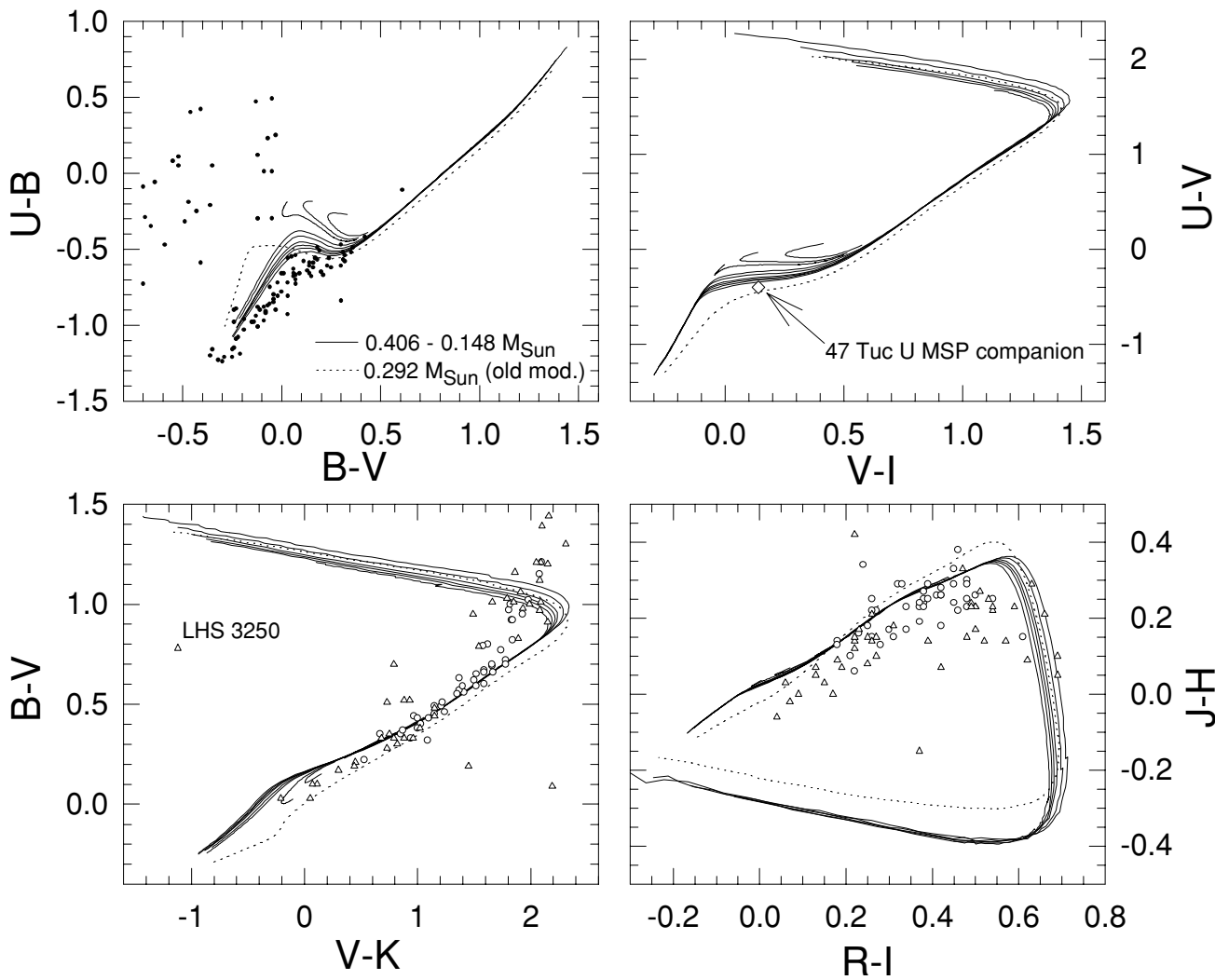

Figure 9. Colour-colour diagrams for sequences corresponding to our He WDs with stellar masses 0.406, 0.360, 0.327, 0.292, 0.242, 0.196, 0.169 0.160 and $0.148 \mathrm{M}_{\odot}$ (solid lines). For comparison, we display the old $0.292-\mathrm{M}_{\odot}$ model sequence (dotted line). Filled circles in the $(U-B, B-V)$ diagram correspond to a sample of seemingly single DA (hydrogen-line) and DB (helium-line) WDs from Saffer et al. (1998). The lower diagrams contain observations of DA (open circles) and non-DA (open triangles) WDs from Bergeron et al. (2001). The 47 Tuc U MSP companion (denoted by 'U') (Edmonds et al. 2001) and LHS 3250 (Harris et al. 1999) are shown in the $(U-V, V-I)$ and $(B-V, V-K)$ diagrams, respectively.

the evolution following the end of mass transfer episodes markedly depends upon the details of how the envelope was lost, we have attempted here a more physically-sound treatment to obtain more realistic initial He WD models than that assumed in Serenelli et al. (2001). Specifically, the WD progenitor was supposed to be in a binary system with a $1.4-\mathrm{M}_{\odot}$ companion (representative of a neutron star) and, during pre-WD evolution, mass-loss rates were adjusted so as to keep the radius of the progenitor close to the Roche lobe radius. In order to compute the mass-loss rates, angular momentum losses due to magnetic braking, gravitational wave emission and mass loss were taken into account (see Sarna, Ergma \& Antipova 2000). In broad outline, the evolution of the 0.148 - and $0.160-\mathrm{M}_{\odot}$ He WD models is qualitatively similar to that of the lowest stellar mass model analysed in Serenelli et al. (2001). Indeed, they are characterized by the absence of hydrogen thermonuclear flashes even when element diffusion is allowed to operate. The lack of thermonuclear flashes forces the evolution of the star to be dominated by stable nuclear burning over most of the entire evolution of these models. As a result, they are characterized by very-long cooling ages. Another resemblance to the lowest-mass models of Serenelli et al. (2001) is the fact that although diffusion proceeds much more slowly than in more massive models, the outer layers become pure hydrogen on time-scales shorter than evolutionary time-scales. The evolution of these very-low-mass He WD models is detailed in Table 4 and in the colour-magnitude diagrams depicted in Figs 10 and 11 .
Observational data of the He WD star detected, as companion to a millisecond pulsar (MSP), in 47 Tucanae by Edmonds et al. (2001) is included in Figs 10 and 11, where this star is denoted by ' $U$ '. From the $\left(M_{\mathrm{V}}, U-V\right)$ diagram we infer a mass slightly higher than $0.17 \mathrm{M}_{\odot}$ for this star, and from the $\left(M_{\mathrm{V}}, V-I\right)$ diagram a value somewhat lower than $0.16 \mathrm{M}_{\odot}$. The pulsar characteristic age of $2 \mathrm{Gyr}$ (see Edmonds et al. 2001, and references cited therein) is consistent with the age derived for the WD from our theoretical models, particularly if the low mass value $\left(0.16 \mathrm{M}_{\odot}\right)$, which yields $2-2.5 \mathrm{Gyr}$, is adopted. A lower value for the age is obtained for a $0.17-\mathrm{M}_{\odot}$ model, resulting in a WD younger than its MSP companion. Evolution time-scales for these low-mass He WDs that do not suffer from thermonuclear flashes (Althaus et al. 2001), however, are dominated by hydrogen burning and in this regard we must recall that metallicity of the WD progenitor can play an important role. He WD models presented in this work were derived from $Z=0.02$ progenitors and, given that $[\mathrm{Fe} / \mathrm{H}]=-0.76$ for 47 Tuc, caution must be taken when trying to infer the age for this He WD with the present models.

In Fig. 11 we also show the six He WD candidates found in NGC 6397 by Taylor et al. (2001). Absolute magnitudes were obtained by using $(m-M)_{\mathrm{V}}=12.29$ and $V-I$ was dereddened by using $E(B-V)=0.18$ and extinction laws for WFPC2 filters given by Holtzman et al. (1995). The three brightest stars of the observed sequence are located between our 0.169 - and $0.196-\mathrm{M}_{\odot}$ evolutionary sequences in Fig. 11, suggesting that these stars could be 0.18$0.1 \mathrm{M}_{\odot} \mathrm{He}$ WDs, whilst the three dimmest ones of that sequence 


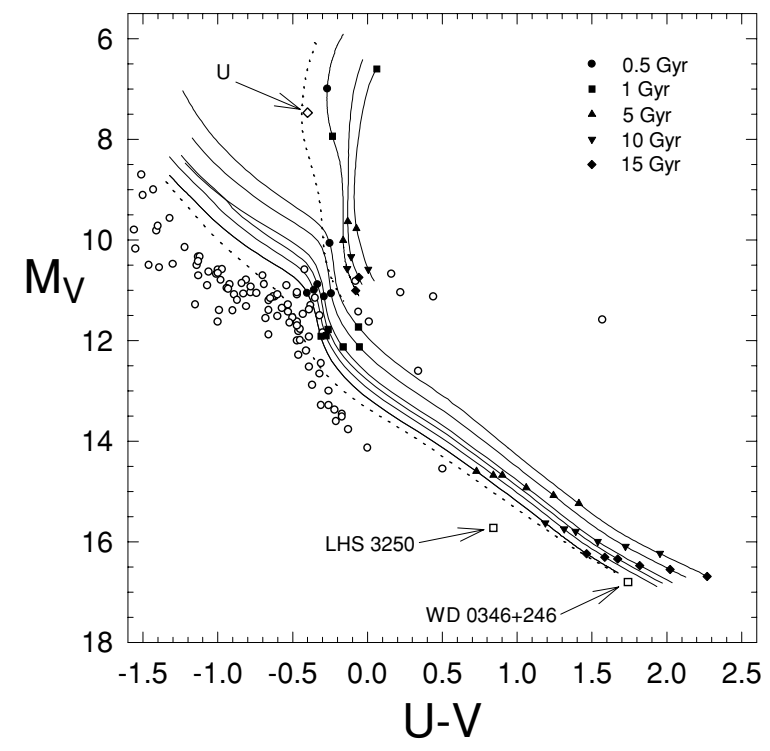

Figure 10. The $\left(M_{\mathrm{V}}, U-V\right)$ magnitude-colour diagram. From the top-right corner, solid lines correspond to He WD sequences with $0.148,0.160,0.169$, $0.196,0.242,0.292,0.327,0.360$ and $0.406 \mathrm{M}_{\odot}$. Dotted lines display our old models with 0.169 and $0.406 \mathrm{M}_{\odot}$. Open circles denote a sample of apparently single DA (hydrogen-line) and DB (helium-line) WDs from Saffer et al. (1998). The 47 Tuc U MSP companion (denoted by 'U') and the ultracool WDs LHS 3250 and WD $0346+246$ are also displayed on the plot.

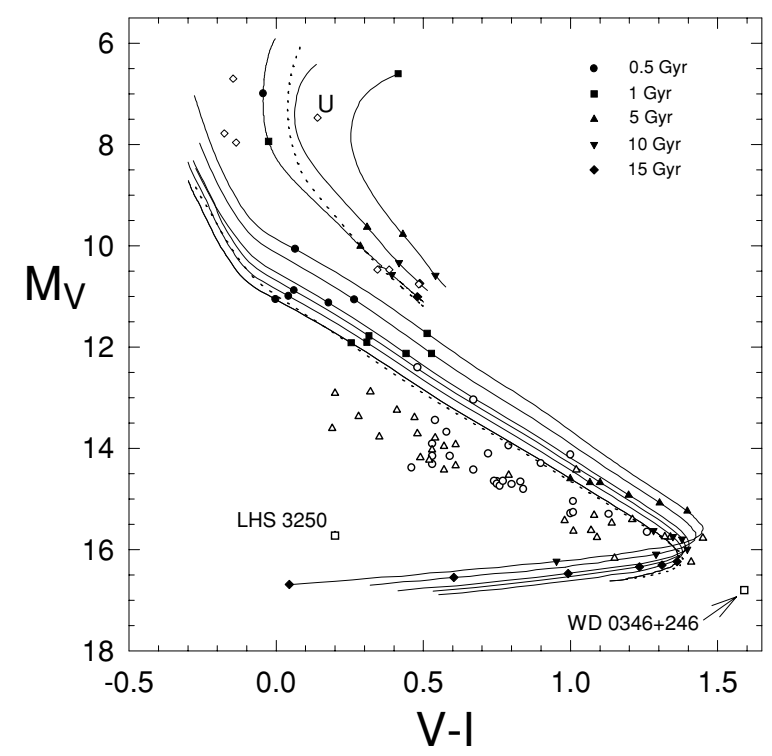

Figure 11. Same as Fig. 10 but for the colour index $V-I$. A sample of cool DA (open circles) and non-DA (open triangles) WDs is included in the figure. Open diamonds show candidate He WDs observed by Taylor et al. (2001) and the 47 Tuc U MSP companion (denoted by 'U').

are compatible with He WDs with stellar masses between 0.16 and $0.17 \mathrm{M}_{\odot}$. Another interesting aspect of our sequences are the ages involved. According to our predictions, the age for the three brightest stars would be $\sim 1$ Gyr. On the other hand the dimmest stars seem to be very old objects, older than $8 \mathrm{Gyr}$ in all cases and even 10 Gyr for the dimmest one. NGC 6397 is a very metal-poor globular cluster $(\mathrm{Fe} / \mathrm{H}=-1.95)$, thus models with a much lower metallicity would help give a more detailed understanding of the observations, since both mass and age determinations could be affected (e.g. the mass threshold for the ocurrence of thermonuclear flashes is a function of the metallicity of the progenitor, thus affecting age determinations even if the WD mass is known). In this regard work is in progress to provide a full grid of He WD models for different metallicities (Serenelli et al. 2002). Recently, Townsley \& Bildsten (2002) have suggested that the three dimmest He WD candidates reported by Taylor et al. could be CO WDs that accrete matter from a $0.15-\mathrm{M}_{\odot}$ main sequence companion at an average rate of $10^{9}-$ $10^{10} \mathrm{M}_{\odot} \mathrm{yr}^{-1}$. Additional observations will be necessary to disentangle this issue.

\section{CONCLUSIONS}

We have presented new computed spectra, colour-magnitude and colour-colour diagrams for the cooling sequences of helium-core white dwarf (WD) stars. These models include more detailed physics entering the atmosphere calculations. Non-ideal effects were carefully taken into account in the computation of the chemical equilibrium of mixed hydrogen and helium gases, using the occupation formalism of Hummer \& Mihalas (1988). We also included a modified version of the optical simulation of Däppen et al. (1987) to compute opacities in non-ideal gases. From these improvements and the evaluation of line opacity from Lyman, Balmer and Pashen series, we determine more realist atmosphere models of He WDs at both early and advanced stages of cooling.

Results from our earlier He WD calculations are confirmed, but a detailed comparison reveals some important quantitative differences. A clear advantage of the newer treatment is the improved behaviour of the emergent flux, especially in hot WDs $\left(T_{\text {eff }}>8000 \mathrm{~K}\right)$, where the line blanketing and Balmer pseudo-continuum process yields substantial differences in ultraviolet and optical regions as compared with our previous calculations, affecting colours in $U B V$ photometry. The use of a non-ideal gas model and up-to-date collision-induced opacities has also allowed a more reliable calculation of cool WD spectra $\left(T_{\text {eff }}<4000 \mathrm{~K}\right)$.

We extended our He WD model grid by computing cooling sequences for stellar masses of 0.148 and $0.160 \mathrm{M}_{\odot}$. Comparison of the colours evaluated for our sequence of models with recent observational data from He WD candidates in the cluster NGC 6397 reported by Taylor et al. (2001), and the He WD companion to a millisecond pulsar in 47 Tucanae observed by Edmonds et al. (2001), allow us to infer low mass values for these objets (ranging from 0.16 up to $\left.0.19 \mathrm{M}_{\odot}\right)$. In the case of 47 Tuc, our age prediction for the He WD (1-2.5 Gyr) agrees reasonably well with the pulsar characteristic age ( $2 \mathrm{Gyr})$. However, given the low metallicity of these clusters, a grid of He WD models with low-metallicity progenitors is needed in order to contrast these and future observations with model predictions.

With the present extensive and physically-sound grid of He WD, we provide a better understanding of the spectral evolution of lowmass WDs. Our resulting He WD models are particularly useful for interpretive data of very low-mass WDs within the current detectability limit, allowing to determine their physical parameters (mass, age, temperature), and to constrain those of their possible binary companions.

Detailed tabulations of the results presented here are available at http://www.fcaglp.unlp.edu.ar/ serenell or upon request from the authors at their e-mail addresses. 


\section{ACKNOWLEDGMENTS}

We are grateful to P. Bergeron and D. Saumon for informative collaboration regarding Table 3. We also thank M. Barstow, our referee, for useful comments that improved the original version of our paper.

\section{REFERENCES}

Althaus L. G., Serenelli A. M., Benvenuto O. G., 2001, MNRAS, 323, 471 Anderson L. S., 1989, ApJ, 339, 558

Benvenuto O. G., Althaus L. G., 1998, MNRAS, 293, 177

Bergeron P., 1988, PhD thesis, Université de Montréal

Bergeron P., 2001, ApJ, 558, 369

Bergeron P., Wesemael F., Fontaine G., 1991, ApJ, 367, 253

Bergeron P., Saffer R. A., Liebert J., 1992, ApJ, 394, 228

Bergeron P., Saumon D., Wesemael F., 1995, ApJ, 443, 764

Bergeron P., Ruiz M. T., Leggett S. K., 1997, ApJS, 108, 339

Bergeron P., Leggett S. K., Ruiz M. T., 2001, ApJS, 133, 413

Böhm H. K., Carbon T. R., Fontaine G., Van Horn H. M.,1977, ApJ, 217, 521

Borysow A., Jorgensen U. G., Zheng C.,1997, A\&A, 324, 185

Borysow A., Jorgensen U. G., Fu Y., 2001, JQSRT, 68, 235

Bragaglia A., Greggio L., Renzini A., D’Odorico S., 1990, ApJ, 365, L13

Bragaglia A., Renzini A., Bergeron P., 1995, ApJ, 443, 735

Cool A. M., Grindlay J. E., Cohn H. N., Lugger P. M., Bailyn C. D., 1998, ApJ, 508, L75

Däppen W., Anderson L., Mihalas D., 1987, ApJ, 319, 195 (DAM)

Derrider G., Van Rensbergen W., 1976, A\&AS, 23, 147

Driebe T., Schönberner D., Blöcker T., Herwig F., 1998, A\&A, 339, 123

Edmonds P. D., Grindlay J. E., Cool A. M., Cohn H. N., Lugger P. M., Bailyn C. D., 1999, ApJ, 516, 250

Edmonds P. D., Gilliland R. L., Heinke C. O., Grindlay J. E., Camilo F., 2001, ApJ, 557, L57

Graboske H. C., Jr., Harwood D. J., Rogers F. J., 1969, Phys. Rev., 186, 210

Greenstein J. L., 1986, ApJ, 304, 334

Griem H. R., 1960, ApJ, 132, 883

Gustafsson M., Frommhold L., 2001, ApJ, 546, 1168

Hansen B. M. S., Phinney E. S., 1998, MNRAS, 294, 557

Harris H. C., Dahn C. C., Vrba F. J., Henden A. A., Liebert J., Schmidt G. D., Reid I. N., 1999, ApJ, 524, 1000

Hayes D. S., 1985, in Hayes D. S., Pasinetti L. E., Davis Philip A. G., eds, IAU Symp. 111, Calibration of Fundamental Stellar Quantities. Dordrech, Reidel, p. 225

Herzberg G., 1950, Molecular Spectra and Molecular Structure I, Spectra of Diatomic Molecules. 2nd edn, Van Nostrand, Princeton

Holtzman J. A., Burrows C. J., Casertano S., Hester J. J., Trauger J. T., Watson A. M., Worthey G., 1995, PASP, 107, 1065
Hubeny I., Hummer D. G., Lanz T., 1994, A\&A, 282, 151 (HHL)

Hummer D. G., Mihalas D., 1988, ApJ, 331, 794

Jorgensen U. G., Hammer D., Borysow A., Falkesgaard J., 2000, A\&A, 361, 283

Kapranidis S., 1983, ApJ, 275, 342

Kurucz R. L., 1979, ApJS, 40, 1

Landau L. D., Lifshitz E. M., 1980, Course of Theoretical Physics V, Statistical Physics. 3rd edn, Part 1. Pergamon, London

Lenzuni P., Saumon D., 1992, Rev. Mex. A. A., 23, 223

Marsh T. R., 1995, MNRAS, 275, L1

Marsh T. R., Dhillon V. S., Duck S. R., 1995, MNRAS, 275, 828

Martin W. C., 1973, J. Phys. Chem. Ref. Data, Vol. 2, No. 2, 257

Mazzitelli I., 1989, ApJ, 340, 249

Mihalas D., 1966, ApJS, 13, 1

Mihalas D., Däppen W., Hummer D. G., 1988, ApJ, 331, 815

Moran C., Marsh T. R., Bragaglia A., 1997, MNRAS, 288, 538

Nayfonov A., Däppen W., Hummer D. G., Mihalas D., 1999, ApJ, 526, 451

Oppenheimer B. R. et al., 2001, ApJ, 550, 448

Orosz J. A., Wade R. A., Harlow J. J. B., Thorstensen J. R., Taylor C. J., Eracleous M., 1999, AJ, 117, 1598

Robnik M., Kundt W., 1983, A\&A, 120, 227

Rohrmann R. D., 2001, MNRAS, 323, 699

Ross M., Ree F. H., Young D. A., 1983, J. Chem. Phys. 79(3), 1487

Saffer R. A., Livio M., Yungelson L. R., 1998, ApJ, 502, 394

Sarna M. J., Antipova J., Ergma E., 1999, in Solheim J.-E., Meistas E. G., eds, ASP Conf. Ser. Vol. 169, 11th European Workshop on White Dwarfs. p. 400

Sarna M. J., Ergma E., Antipova J., 2000, MNRAS, 316, 84

Saumon D., Chabrier G., 1991, Phys. Rev. A, 44, 5122

Saumon D., Jacobson S. B., 1999, ApJ, 511, L107

Saumon D., Chabrier G., Van Horn H. M., 1995, ApJS, 99, 713

Saumon D., Chabrier G., Wagner D. J., Xie X., 2000, High Press. Res., 16, 331

Serenelli A. M., Althaus L. G., Rohrmann R. D., Benvenuto O. G., 2001, MNRAS, 325, 607

Serenelli A. M., Althaus L. G., Rohrmann R. D., 2002, MNRAS, submitted

Taylor J. M., Grindlay J. E., Edmonds P. D., Cool A. M., 2001, ApJ, 553, L169

Townsley D. M., Bildsten L., 2002, ApJ, 565, L35

van Kerkwijk M. H., Bell J. F., Kaspi V. M., Kulkarni S. R., 2000, ApJ, 530, L37

Vardya M. S., 1965, MNRAS, 129, 25

Vidal C. R., Cooper J., Smith E. W., 1973, ApJS, 25, 37

Weeks J. D., Chandler D., Andersen H. C., 1971, J. Chem. Phys., 54, 5237

Weise W. L., Kelleher D. E., Paquette D. R., 1972, Phys. Rev. A, 6, 1132

This paper has been typeset from a $\mathrm{T}_{\mathrm{E}} \mathrm{X} / \mathrm{L} \mathrm{T} \mathrm{E} \mathrm{X}$ file prepared by the author. 